\title{
La sociedad y la cultura andina contemporánea: estudio de los saberes para la salud y la enfermedad en los pueblos originarios del norte de Chile
}

\author{
POR \\ Vivian Gavilán. Universidad de Tarapacá / Patricia Vigueras, Michel Parra. \\ Universidad Arturo Prat / Carlos Madariaga. Universidad \\ de Chile / Nicolás Morales. CIESAS / Alejandra Arratia. Taller \\ de Estudios Andinos / Rosa Andrade. Taller de Estudios Andinos
}

A J. y S. (Q.E.P.D.) por su amistad e invitación a la reflexión sobre la vida y la muerte, relación inacabada, lugar donde las diferencias se pierden en la camanchaca del amanecer andino

El estudio de los saberes para la salud y la enfermedad y de las prácticas médicas en la población de origen indígena plantea desafíos teóricos, sociales y políticos. Desde el punto de vista del análisis, la heterogeneidad cultural en la que se encuentra obliga a considerar los procesos de modernización, lo cual implica asumir una perspectiva histórica que no siempre consideran las investigaciones sobre la sociedad y cultura andina contemporánea. Este artículo pretende avanzar en este sentido. Planteamos que el peso de la escolaridad es gravitante en la diferenciación social y cultural y por lo tanto el nivel alcanzado incide en la comprensión de la naturaleza del cuerpo y su funcionamiento y en las prácticas para la salud. Los datos coinciden en gran parte con los informados por otros investigadores; a partir de estos, proponemos que es preciso ampliar el debate sobre la cuestión de la materialidad-inmaterialidad de las bases teóricas del pensamiento médico andino.

Palabras Clave: Sociedad y cultura andina contemporánea; medicina andina; conocimiento indigena; salud y pueblos originarios. 


\section{INTRODUCCIÓN}

Nuestro interés por el estudio de las prácticas médicas en la población de origen indígena en la región de Tarapacá partió de un hecho al cual nos enfrentamos en diciembre de 2004. Se trató de un caso problemático para el servicio de siquiatría del Hospital E. Torres G. de Iquique. Los diagnósticos elaborados para explicar y o comprender la enfermedad de un joven de 18 años (S.), residente en Pozo Almonte, pequeña ciudad del desierto tarapaqueño, y con padres provenientes de las comunidades de la zona altiplánica, diferían notablemente entre la familia y los equipos de salud. Los padres y abuelos argumentaron que estaba agarrado por Seren T'alla, entidad demoníaca (supay) a la cual se le pide el don de la armonía musical y cuya morada es una vertiente. Es frecuente que las personas de estas comunidades consideren que la biomedicina ${ }^{1}$ no tiene eficacia para sanar esta enfermedad, que en lengua aymara se nombra katjata, ¿Por qué, entonces, decidieron concurrir a la posta local, a la clínica privada y al hospital en busca de sanidad? De manera alternativa, acudieron a la medicina indígena. Después de varias semanas sin mejoría, la familia tomó la decisión de llevarlo a casa para compartir sus últimos días. A fines de enero de 2005, S. muere. La única interpretación que tuvimos de este acontecimiento fue que ninguno de los sistemas médicos, en los cuales confió esta familia, pudo salvar la vida de S., recién graduado de enseñanza media.

El caso planteó y plantea un desafío a las ciencias sociales, a las ciencias de la salud y obviamente, a los planificadores en salud pública. Una de las interrogantes que formulamos fue ¿cómo se articulan modelos médicos tan diferentes como son el biomédico y el basado en la religiosidad andina? El proceso de investigación, con enfoque cualitativo, nos llevó a indagar sobre los fundamentos ontológicos y epistémicos del proceso salud-enfermedad-atención de las personas que se adscriben a los pueblos originarios. Adelantamos aquí avances acerca de los saberes y representaciones del funcionamiento del cuerpo y de las prácticas médicas, en un contexto de gran heterogeneidad cultural $\mathrm{y}$ en un momento en el que los mismos parecen ser reemplazados $-\mathrm{o}$ al menos resignificados - por la biología. Nos pareció importante definir un marco

1 «Biomedicina [Biomedicine o Western-medicine]: también llamada medicina occidental, científica, alopática, cosmopolita o etnomedicina fisiológicamente orientada. Designa al sistema médico desarrollado en Occidente desde la Ilustración y que se caracteriza por asumir la distinción cartesiana entre cuerpo y mente y por su comprensión de las enfermedades en tanto que entidades definidas producidas por causas únicas. Ver Modelo Médico Hegemónico». Comelles y Martínez, 1993:85. 
teórico que facilitara la comprensión de las dinámicas históricas a fin de abordar tanto las continuidades como las discontinuidades culturales en la medicina indígena. Como ha señalado E. Menéndez, ello es importante, mas no suficiente. Intentamos asumir un concepto de salud que reconozca su múltiple dimensionalidad y que se oriente a buscar soluciones concretas al abatimiento/no abatimiento de los daños a la salud, dadas las condiciones sanitarias desiguales en la que se encuentra la población de origen indígena, en Tarapacá y en el país ${ }^{2}$.

Entre los resultados obtenidos se halla el gran peso que adquiere la escolaridad en la heterogeneidad al interior de la colectividad que se adscribe étnicamente al pueblo aymara. Aquellas personas que alcanzaron mayores niveles presentan más apego a la biología como marco referencial para comprender la ontología del cuerpo humano. En consecuencia, menor importancia de los conocimientos y acciones para la salud y la enfermedad transmitidas por las generaciones anteriores y mayor acercamiento a la biomedicina. Otro hallazgo relevante es que la adscripción religiosa y la influencia de los medios de comunicación son también factores que inciden en una mayor o menor influencia del saber oficial del cuerpo y la salud, pero éstos son menos importantes que la escolaridad.

Como veremos, las representaciones del proceso salud-enfermedad-atención se basan en un conjunto de relaciones entre lo empírico, una lógica y una racionalidad que se debe investigar. Observamos que en las personas con menor escolaridad las prácticas médicas siguen una racionalidad basada en conceptos cuyos contenidos se orientan a comprender la naturaleza del cuerpo humano en su materialidad e inmaterialidad. Su estructura orgánica operaría sobre procesos que van más allá de lo estrictamente biológico.

Por otra parte, los datos muestran que el sistema médico indígena se encuentra en una situación crítica porque los procesos de dominación económica y cultural imponen un proyecto civilizatorio que tiende a valorar la biomedicina y a subvalorar los conocimientos y prácticas médicas que han restaurado la salud de las colectividades étnicas y que podrían contribuir a resolver problemas de salud en otros grupos sociales.

Exponemos en primer lugar, los antecedentes metodológicos de la investigación; sigue una revisión crítica de los estudios acerca de la medicina andina a fin de posicionarnos en el debate. En tercer lugar, realizamos una evaluación de la situación de las prácticas médicas en la región que avalan la expansión de los saberes de la medicina moderna. El cuarto apartado se refiere a los sabe-

\footnotetext{
2 Menéndez, 7 (México D.F., 1994): 74.
} 
res y representaciones que muestran continuidades culturales y la especificidad de las prácticas médicas de origen indígena las que se asemejan a la data etnográfica referida a comunidades de Bolivia y Perú. El quinto y último tópico intenta unir los fragmentos para recuperar la forma de concebir la vida.

\section{ANTECEDENTES METODOLÓGICOS DE LA INVESTIGACIÓN}

Entre los años 2006 y $2008^{3}$ iniciamos el trabajo en la comuna de Alto Hospicio $^{4}$, distinguiendo cuatro colectividades a partir de los datos sobre migraciones entregados por el censo 2002: aymara (chilenos), inmigrante transfronterizo (peruanos y bolivianos), colectividad del centro y sur del país y nortinos no indígenas (Arica-Ovalle). Entrevistamos a 49 personas, 11 de ellas se adscribieron étnicamente al pueblo aymara, con diversos niveles de escolaridad (de educación básica incompleta a universitaria completa), con adscripción a las iglesias católica y pentecostal y con residencia más estable en la ciudad. Durante la primera mitad de 2009 continuamos con una corta investigación acerca de los saberes médicos a fin de rescatar el patrimonio cultural en salud de los pueblos originarios en Tarapacá ${ }^{5}$. En este caso entrevistamos a 14 personas residentes o multiresidentes en las comunas de la Provincia del Tamarugal y en la comuna de Alto Hospicio. Sólo dos de ellas cuentan con enseñanza media completa técnica profesional, en tanto que las restantes cursaron enseñanza básica. Seis ofician como sanador-a (qolliri-yatiri), tres se desempeñan como promotoras de salud y cinco hacen uso frecuente de este tipo de atención médica. Según adscripción étnica, diez dicen formar parte del pueblo aymara, tres declaran pertenecer al pueblo chipaya y una se adscribe al pueblo quechua.

Aunque las pautas de entrevistas no fueron las mismas en ambas investigaciones se incluyeron dos apartados en común: representaciones del proceso salud-enfermedad-atención y prácticas de autoatención ${ }^{6}$. Una de las modalidades

3 Proyecto Fondo de Investigación en Salud (FONIS-CONICYT): «Estudio y funcionamiento del sistema de atención primaria de la comuna de Alto Hospicio desde un enfoque intercultural» 2006.

4 Se trata de un territorio recientemente poblado y declarado comuna, unidad mínima de la administración política del país. Residen allí, en medio del desierto, 80.000 personas aproximadamente, en su mayoría inmigrantes.

5 Proyecto FONDART «Suma Kamaña»: estudio y valoración de las prácticas de salud y enfermedad en las comunidades de origen indígena en la Provincia del Tamarugal, 2009.

${ }^{6}$ Por prácticas de autoatención entendemos aquellas formas de atención diversas que los sujetos y grupos sociales no sólo usan, sino que las sintetizan, articulan, mezclan o yuxtaponen. 
de recolección de datos más ricas sobre los saberes para la salud y la enfermedad fue la realización de un encuentro de sanadores en el que aplicamos entrevistas colectivas y conversaciones fluidas. La participación en éste de sanadores y usuarios chipaya, aymara, de la zona de valles y del altiplano y residentes también en las ciudades, ofreció un espacio de intercambio y debate de gran interés para el conocimiento del sistema médico andino y para evaluar la posición subalterna en la que se encuentra.

\section{LOS ESTUDIOS DE LA MEDICINA ANDINA}

Gundermann y González ${ }^{7}$ afirman que la medicina andina o el conjunto de conocimientos, prácticas, actores y sistemas médicos en el norte grande de Chile podría ser uno de cuatro temas de un posible programa de investigaciones sobre la cultura andina regional, dirigido a llenar un vacío actualmente existente en este ámbito. ¿Por qué privilegiar este aspecto de la vida de las familias de origen indígena para conocer la cultura andina en este territorio? Argumentan que se trataría de prácticas culturales institucionalizadas que habrían mantenido una notable continuidad, aun cuando éstas se han transformado y complejizado, mantendrían una considerable fidelidad cultural. Los autores no justifican por qué estas prácticas expresarían continuidades sobre otros campos de estudio, tales como los procesos de socialización, relaciones familiares, etc.

María Ester Grebe propone la existencia de dicha continuidad y estabilidad en los modos de observar, conocer y representar el mundo que rodea a los pueblos originarios. Sugiere que sus patrones cognitivos y simbólicos persisten aún después de cuatro siglos de conquista y colonización a pesar de factores geográficos, históricos, sociales y lingüísticos que inciden en la generación de influencias contrarias, las cuales podrían haberlos debilitado o destruido. Estos patrones habrían sobrevivido más allá del debilitamiento, hibridación o extinción de sus propias lenguas indígenas: serían supraligüísticos. De este modo, en el cam-

Se funda en una concepción de prácticas de sanación desde la experiencia social de los grupos humanos y no de sujetos aislados; resalta la dimensión de lo colectivo en tanto escenario relacional que produce formas concretas de intervención individual, más o menos características de cada grupo, para abordar el «padecimiento» y no sólo la enfermedad al interior de los microgrupos familiares. En este sentido, «la autoatención constituye no solo la forma de atención más constante sino el principal núcleo de articulación práctica de las diferentes formas de atención, la mayoría de las cuales no pueden funcionar completamente si no se articula con el proceso de autoatención» (Menéndez, 2003: 190-191).

7 Gundermann y González, 41/1 (Arica, 2009): 134 
po de la medicina aymara, esta autora sostiene que estaría unida a conocimientos, creencias y prácticas que denotan gran antigüedad ${ }^{8}$. Una posición radical la hallamos entre quienes sostienen que existiría un sistema de salud aymara y un sistema occidental cosmopolita o moderno en franca oposición y que la vigencia del primero sería una prueba de la resistencia cultural de los andinos frente al sistema biomédico. Existirían diferencias que dan lugar a choques culturales entre dos sistemas de salud ${ }^{9}$. J. van Kessel, quien se ha interesado especialmente en este tema, ha argumentado que el sistema económico y cultural dominante socava permanentemente los saberes y tecnologías médicas de los aymaras; aun así, persistiría la unidad cuerpo-cosmos. Para este autor, el ayllu y la familia representa la concreción del cosmos andino por lo cual su estudio es importante para entender la concepción de salud-enfermedad y el ritual terapéutico. Sostiene que la enfermedad era y sigue siendo importante no por sí misma sino por su significación espiritual, trascendental; tendría tres dimensiones: la ética, la religiosa y la social. Las causas de la enfermedad y, por lo tanto, el método de curar, se encuentran en lo espiritual y lo sobrenatural. Aunque en su nosología habría que pensar en enfermedades somáticas, psicosomáticas, endémicas y epidémicas, en su terapia hay que incluir no sólo los fármacos, dietas y demás elementos de una tecnología médica positiva y empírica, sino también su ambientación social, su montaje ritual, sus elementos sicológicos y su sistema comunicativo que incluye a curandero, enfermo, parientes y divinidades ${ }^{10}$. Guerrero sigue la misma perspectiva, a través del estudio del acto médico en su dimensión simbólica y compara la medicina andina (expresada en el agente principal, yatiri en lengua aymara) con la medicina pentecostal (expresada en su líder, el pastor). La continuidad de la tradición se observaría en los elementos simbólicos de la curación de las enfermedades, pues concurren elementos míticos-religiosos derivados de una particular interpretación de la Biblia. En tanto que la ruptura se observaría en diferencias basadas en horizontes culturales e ideológicos distintos, las que se plantean en el ámbito de la etiología, la formación de los agentes y la naturaleza de los tratamientos (el pastor utiliza la Biblia, el yatiri, utiliza hierbas). Concluye en que el estudio comparativo entre ambos agentes muestra que se trata de una transición de una nueva praxis médica, pero praxis que tiene sus raíces en la cultura andina ${ }^{11}$.

Se ha propuesto, también, que los andinos combinan su propio sistema médico con la medicina moderna, conviviendo paralelamente o articulándose allí

\footnotetext{
8 Grebe Vicuña, 10 (Arica, 1983): 163; 1998: 73-74.

9 Arnold y otros, 1999: 17.

10 Kessel, 10 (Arica, 1983): 24; 13 (Iquique, 1985): 9.

11 Guerrero Jiménez, XXVII/2 (Arica, 1995): 164.
} 
donde son compatibles. Chamorro y Tocornal estudian la medicina atacameña y ratifican la idea de Kessel y Guerrero al postular que la religiosidad cumple un rol fundamental en el itinerario terapéutico ${ }^{12}$. La salud se restituye a partir de la fe, misma que estaría presente en el uso de la medicina moderna. La fe sería el elemento que vincula a la biomedicina con el modelo propiamente atacameño. Siguiendo la propuesta de Foster, las autoras sugieren que ambos modelos coincidirían en la concepción de las enfermedades «naturalistas» basadas en el modelo hipocrático del cuerpo y el entorno. Concluyen que la ontología del cuerpo y del ambiente se encuentra más cerca de la relación con el medio y las tradiciones propias del mundo andino que de la conceptualización de la vida y la muerte presente en el modelo médico basado en la biomedicina. En tanto que las enfermedades «personalistas y emocionales» se tratan preferentemente por la medicina tradicional atacameña, debido al componente mágico-religioso que involucra el tratamiento ${ }^{13}$.

Del mismo modo, G. Fernández encuentra en las comunidades de Bolivia una cosmovisión andina de la salud que convive con la biomedicina. Es indicada por los yatiris cuando éstos comprenden que no pueden tratarlas y deben ser derivadas al médico. Postula que para los aymaras el objeto de atención no es el paciente o el órgano enfermo aislado del resto del cuerpo y de sus relaciones sociales y familiares, al contrario, le interesa la persona íntegra, física, social, cultural, en consonancia con la memoria histórica y con sus relaciones comunitarias. Las enfermedades se producen por el descontento de los seres tutelares hambrientos a los que no se les ha alimentado con el ayni culinario, mesas, rituales, libaciones bajo el principio de reciprocidad. Se producen enfermedades por estar en lugares fuertes o en malas horas (saxra ura) ${ }^{14}$.

Con algunos matices, la mayoría de los autores exponen datos etnográficos similares, sin embargo difieren en sus interpretaciones. Vemos, por una parte, cierta tendencia a enfatizar en la permanencia de principios precolombinos, olvidando los cambios y los efectos de los procesos de colonización cultural en el que las comunidades indígenas se inscriben. Un ejemplo de las discontinuidades es la existencia del ayllu como entidad corporativa que agrupa a varias

12 Se entiende por itinerario terapéutico aquel patrón de búsqueda de atención que remite a la trayectoria que un individuo o grupo social realiza con el fin de dar respuesta a una demanda de salud. Diversos autores han empleado diferentes términos para dar cuenta de estas estrategias: carrera curativa (Freidson, 1978), conductas de búsqueda de atención (Kleinman, 1980: 52), itinerario terapéutico (Citarella, 1995: 515), entre otros.

13 Chamorro y Tocornal, 30 (San Pedro de Atacama, 2005). Las autoras se basan en la clasificación propuesta por Foster \& Anderson (1978).

14 Fernández Juárez, 28 (Madrid, 1998): 259-282. 
unidades domésticas. En el caso de las comunidades históricas del norte chileno, si bien se halla presente en la memoria, no tiene existencia real y no cumple funciones a nivel de la organización. Los cambios ocurridos han llevado a un incremento de la autonomía y el poder de las unidades domésticas y los grupos correspondientes a las líneas parentales más inmediatas respecto del conjunto de la comunidad ${ }^{15}$. En relación a las formas de autoatención en salud, el uso creciente de medicinas de la industria farmacéutica y en el orden de los fundamentos epistémicos la incidencia de los procesos de secularización generados por el aumento de las tasas de escolarización.

Por otra parte, la literatura muestra cierta tendencia a estudiar el fenómeno de la salud en su dimensión simbólica como resultado de investigaciones sobre religiosidad, lo cual resta importancia al estudio de la misma en su dimensión material, que junto a la inmaterial, opera en el concepto de salud en la tradición andina. Esta relación cuestiona la clasificación de las enfermedades en naturalistas y personalistas, ya que ésta tiende a reproducir un dualismo etiológico entre una «naturaleza» y un dominio «mágico-religioso» que reduce cualquier agencia social a un fenómeno mágico y asimila toda idea de «naturaleza» a un modelo humoral hipocrático desconociendo las especificidades de la ontología andina ${ }^{16}$.

Los resultados de esta investigación muestran continuidades y discontinuidades en las prácticas médicas y sus bases teóricas como cualquier otra actividad de los sujetos que hoy se adscriben étnicamente. Con otras palabras, la medicina de los pueblos originarios no difiere de otras prácticas de la vida de los mismos. En realidad, el conocimiento de cualquier sistema médico y sus fundamentos ontológicos y epistémicos depende de los conceptos de sociedad y cultura que asumamos. Frecuentemente, los estudios regionales no explicitan su orientación teórica. Concordamos con González y Gundermann en que ha existido cierta tendencia a deshistorizar la realidad en la que las colectividades étnicas se desenvuelven. Tanto indígenas como no indígenas formamos parte de los procesos sociohistióricos que han ocurrido en este territorio. En este sentido, las transformaciones impulsadas durante los siglos XIX y XX son fundamentales para comprender los procesos étnicos e interétnicos que emergieron con los procesos de modernización y sus efectos en la dinámica cultural ${ }^{17}$.

Siguiendo a Bastide, Cuche nos recuerda que lo cultural no puede estudiarse independientemente de lo social; por lo tanto, las relaciones culturales deben ser

15 Cf. Gundermann Kroll, 2001: 29.

16 Augé, 26/97 (París, 1986): 82-3.

17 Ibidem: 139. 
analizadas en el interior de los diferentes marcos de relaciones sociales, entre las cuales las relaciones de poder son centrales. Para este autor los elementos que componen una cultura - dado que provienen de fuentes diversas en el espacio y en el tiempo - no están nunca integrados unos a otros. Esto abre la posibilidad al cambio, mismo que sería más marcado en contextos de modernización planificada. Es en este contexto que se desliza la libertad de los individuos y de los grupos para manipular la cultura. Por consiguiente, no habría - de un ladoculturas puras y - del otro- culturas mestizas. Todas por el hecho universal de los contactos culturales son «mixtas», hechas de continuidades y discontinuidades. La discontinuidad cultural debería buscarse más en el orden temporal que en el orden espacial. La continuidad afirmada de una cultura dada es, con frecuencia, más ideología que realidad. Y esta pretendida continuidad será tanto más afirmada cuando la discontinuidad estalla en los hechos: en los momentos de ruptura, el discurso de la continuidad es una ideología de la compensación. Cuche propone que a menudo hay mayor continuidad entre dos culturas que están en contacto prolongado que entre los diferentes estados de un mismo sistema cultural tomado en momentos distintos de su evolución histórica ${ }^{18}$. Desde esta perspectiva, en la región no hay un sistema médico andino puro y otro mestizo, menos una «medicina tradicional» $\mathrm{y}$ «una medicina occidental» proveniente de la sociedad occidental como entidad metafísica. La medicina andina se ha apropiado de conceptos y prácticas de la medicina occidental, como efecto de los procesos de colonización. La oposición medicina occidental/medicina indígena simplifica una realidad mucho más compleja. La medicina occidental de los siglos XVI, XVII y XVIII tiene similitudes con la actual medicina andina. Asimismo, el pensamiento médico de los pueblos originarios no se detiene, ha evolucionado. La historia de la medicina de occidente es mucho más que la medicina moderna y la biomedicina sólo es una entre otras aproximaciones epistémicas de la modernidad. Ésta es producto del desarrollo histórico europeo que se expandió en un contexto de relaciones capitalistas de producción que busca extender el conocimiento y la posesión de la naturaleza, la producción, la circulación y el consumo de los bienes motivado preferentemente por el incremento del lu$\mathrm{cro}^{19}$. La población andina ha sabido articular diferentes tipos de propuestas médicas a partir de teorías y praxis mixtas, las que constituyen un acervo cultural de gran valor para la salud de los pueblos.

Este ángulo de mirada coincide con lo señalado por E. Menéndez, esto es, que la «medicina tradicional» no debiera ser analizada «en sí», sino referida al sistema cultural dentro del cual el grupo utiliza un espectro de representacio-

18 Cuche Denys, 2002: 82.

19 Larraín, 66 (Santiago, 1997): 32. 
nes y prácticas producto no sólo de dicha «medicina», sino de un conjunto de saberes que redefinen continuamente el sentido, significado y uso de la misma. Ello implica asumir una perspectiva relacional, es decir, analizar el sistema de representaciones y de prácticas que opera un grupo determinado en su trato con el enfermo, ya sea referido a un padecimiento tradicional como el «empacho» o a una enfermedad definida en términos alopáticos como la gastroenteritis. El autor cita a Cosminsky (1986) para señalar que ello permitiría observar los procesos de síntesis, de yuxtaposición o de exclusión de prácticas y representaciones procedentes de diferentes saberes, pero que, en los conjuntos sociales, se organizan de una determinada manera. Es en la descripción y análisis de estos procesos relacionales que observaremos el lugar que ocupan lo «tradicional» o lo «científico», pero lo haremos en términos del sistema de prácticas y representaciones que está teniendo lugar en la realidad de un enfermo atendido en el hogar, o por una hierbera, o por un médico alópata, o por un espiritualista o, como puede ocurrir, por todos ellos en forma sucesiva ${ }^{20}$.

Menéndez acierta al proponer que las interpretaciones y usos del «saber médico tradicional» deberían ser estudiados en su relación con el proceso salud-enfermedad-atención asumido, y no sólo en términos de continuidad/discontinuidad cultural, sino en términos de abatimiento/no abatimiento de los daños a la salud, dadas las condiciones negativas que operan sobre la salud, la enfermedad y la muerte en los grupos indígenas americanos.

\section{LA SITUACIÓN ACTUAL DE LAS PRÁCTICAS MÉDICAS EN LA POBLACIÓN DE ORIGEN INDÍGENA}

Uno de los resultados de los procesos sociohistóricos ocurridos en Tarapacá es la presencia de tres pueblos originarios: aymara, quechua y chipaya ${ }^{21}$. Se registran dos lenguas: aymara y chipaya, correspondientes a las colectividades étnicas aymara y chipaya. En ambas, se observan nociones similares referentes al proceso salud-enfermedad-atención, que hablan de tradiciones comunes. Ello se refleja en términos lingüísticos compartidos, pero la lengua chipaya ${ }^{22}$

20 Menéndez, 7 (México D.F., 1994).

21 Aunque no contamos con cifras que nos informen sobre el número de población chipaya en Tarapacá, existe un gran número de familias instaladas en las comunas de Alto Hospicio, Camiña, Pozo Almonte, Pica y Antofagasta. Gran parte de las mismas buscan la residencia definitiva, sin embargo continúan con fuertes lazos con su comunidad de origen.

${ }^{22}$ Las lenguas chipaya y aymara han estado históricamente en contacto y por lo tanto se han producido intercambios lingüísticos importantes. El profesor Elías Ticuna propone que la 
considera especificidades evidentes. Sin embargo, la lengua quechua está débilmente representada.

Se cumple la constante que la lengua no agota la cultura y que ésta no es lo mismo que etnicidad. Las personas que se adscriben étnicamente a los pueblos quechua y aymara argumentan distinciones que se expresan en las prácticas religiosas, hábitos de alimentación, etc. y en las historias locales. En tanto las personas adscritas al pueblo chipaya se consideran distantes de los anteriores como grupo étnico y como colectividad nacional ${ }^{23}$.

Como hemos señalado, este estudio así como otros referidos a la religiosidad y a la construcción simbólica del género ${ }^{24}$, muestran que las prácticas médicas y sus fundamentos teóricos dependen menos de la residencia (multiresidencia) urbano-rural que de los niveles de escolaridad alcanzados. De esta manera, quienes tienen doce y más años de estudio tienden a vincularse más con la medicina alopática. Aunque la adscripción religiosa y el acceso a los medios de comunicación inciden también en un mayor uso de la medicina moderna, tienen un peso menor que la escolaridad.

La escuela ha promovido la secularización y la racionalización de la vida social y un individualismo creciente. En Tarapacá la escolarización ha avanzado de manera diferenciada territorialmente. Los últimos en disminuir las tasas de anafalbetismo y aumentar los años de estudio son las comunidades del altiplano. Si bien la mayoría de los hogares se integraron a los mercados regionales durante el siglo XIX, es sólo después de 1950 que la escolaridad se masifica. La institucionalidad de la salud pública fue, y sigue siendo, el otro sistema que actuó como agente estatal de transformación, sin embargo, son escasos los estudios que nos informen sobre el impacto de la acción pública en la vida social y cultural de las comunidades.

Como se verá más adelante, los pueblos indígenas de Tarapacá son poseedores de un vasto conocimiento, tecnologías y agentes especializados sobre los cuales se erige un sistema médico complejo, y, lo que es más importante, se trata de un tipo de medicina que es necesaria para la salud de los mismos.

variante de la lengua aymara de las comunidades altiplánicas sería un aymara «chipallizada», lo que las distinguiría de las comunidades hablantes de Bolivia y de la zona norte (Comunicación Personal, 2005).

23 De acuerdo a nuestros datos, la emergencia del pueblo quechua en Mamiña estaría asociado a conflictos con la dirigencia aymara, especialmente con personas provenientes de las comunidades del altiplano. Al mismo tiempo se observa la afirmación de la identidad local a través de argumentos vinculados a la pasada adscripción peruana de las generaciones anteriores. Proyecto FONDECYT N. ${ }^{\circ} 1050143$ «Identidades sociales en Tarapacá. Conflictos Interétnicos y nacionalismos en la Sociedad Regional Contemporánea» CIHDE-UNAP.

24 Gavilán y Carrasco 2009, 41/1 (Arica, 2009): 103. 
Sin embargo, estos saberes y estas prácticas no son homogéneos en la población que se adscribe étnicamente. En la zona de valles se ha producido un proceso más acelerado de reemplazo del sistema médico propio de los pueblos rurales por la medicina moderna. En estas localidades se concentró la población hasta mediados del siglo XIX y en la mitad del siglo XX la mayoría de las familias de la región de Tarapacá residían en las ciudades de la costa. Es posible observar, por ejemplo, la desaparición paulatina de sanadores. En algunos casos son reemplazados por las nuevas generaciones, pero ahora como profesionales del sistema público de salud; en otros, por sanadores de la zona altiplánica. Se observa así, una disminución del uso de la medicina tradicional. Si bien las comunidades del altiplano tienden a adoptar la medicina moderna siguiendo el mismo proceso, la presencia más nítida de sanadores ejerciendo una praxis cotidiana habla de una mayor vigencia del sistema médico andino. Pero esta tendencia no se detiene. En Isluga, comunidad altiplánica, se observa la desaparición de yatiris mujeres. Esta pérdida paulatina se expresa sobre todo en la desaparición del «chiriguano», sanador que se asemeja al personaje mítico ekeko, que solía caminar por los pueblos ofreciendo sus servicios de sanación. En el mismo sentido, se contaría únicamente con un "waytiri» (de origen chipaya), sanador de alto prestigio provisto de saberes y técnicas de sanación para problemas complejos. En tanto que los chipaya dispersos por el norte grande chileno, tienden a utilizar a sus propios sanadores y el sistema público de salud. Es probable que el número de sanadores esté en franco retroceso y las nuevas generaciones se integran cada vez más a la sociedad moderna.

Otro aspecto visible del desplazamiento del sistema médico andino y reconocido por los sanadores se observa en la atención del parto. Los partos que fueron atendidos en casa hasta fines del siglo XX, hoy tienden a ser atendidos en el hospital. En ello ha habido una clara incidencia del Estado, dada la prohibición del parto domiciliario ${ }^{25}$. Por otra parte, una cuestión crítica es el no uso de las lenguas, el desplazamiento de las mismas por el castellano es un aspecto que juega en contra de la preservación del conocimiento en salud.

25 Durante los años ochenta se instala la primera paramédica en la Comuna de Colchane. Ella tenía por misión promover el control del embarazo y traslado de las mujeres al hospital de Iquique. En esa época, también por primera vez, las familias comenzaron a recibir los subsidios estatales. Ello ayudó a un mayor control. Las mujeres aceptaron el control del embarazo como una forma para acceder al beneficio, pero eludían el traslado a Iquique. Muchas se iban a parir a lugares de pastoreo o se ocultaban en sus casas, bajo candado para engañar a la profesional. Ellas se resistían por miedo a la forma de ser atendidas, que no respetaran su modelo obstétrico, a ser maltratadas y o por el gasto económico que implicaba el traslado a la ciudad. 
Aquellas personas que se relacionan comúnmente con el aparato estatal, han adoptado un concepto de medicina «occidental» que la reduce a la biomedicina. Ello se entiende en el contexto de la lucha de las reivindicaciones étnicas regionales que buscan afirmar las diferencias culturales; pero también por la fuerte incidencia de las acciones del Ministerio de Salud a través del programa de salud y pueblos indígenas. El interés que manifiesta el Estado por la incorporación del sistema médico indígena al sistema público se limita a incorporar a algunos agentes de salud en sus dispositivos rurales. Ello ha sido reconocido como un signo positivo por la comunidad; no obstante, ha significado la posibilidad de ingresos antes no vistos. De aquí emerge cierta competencia por acceder a ellos, a un empleo remunerado. Es legítimo, pero el problema radica en la improvisación de sanadores, lo cual preocupa a la comunidad y no contribuye al reconocimiento del patrimonio cultural en salud. Siguiendo a J. Breilh, en Chile se empieza a tener conciencia de la diversidad cultural, pero en el campo de la salud va más ligada a la visibilización de concepciones terapéuticas alternativas antes que al reconocimiento de la múltiple dimensionalidad de la salud desde una perspectiva epistemológica renovada ${ }^{26}$.

Estos resultados nos permiten afirmar que el sistema médico indígena se encuentra en una situación crítica porque los procesos de dominación económica y cultural imponen un proyecto civilizatorio que tiende a valorar la biomedicina y a subvalorar los conocimientos y prácticas médicas de los pueblos originarios. El sistema educacional es el que incide en el proceso de regresión de las tradiciones médicas indígenas. A mayor escolaridad, mayor peso de la biología en la ontología del cuerpo humano, menor importancia de los conocimientos y acciones médicas de las generaciones anteriores y en consecuencia mayor acercamiento a la medicina moderna.

El estudio comparativo entre grupos que se adscriben étnicamente y aquéllos que declaran no descender de pueblos originarios con los mismos niveles de escolaridad, indica que los conocimientos y praxis médica de los primeros tienden a ser diversas y ricas en conocimientos de herbolaria, de minerales y sustancias provenientes de animales propias de la región. En tanto que los segundos exponen conocimientos y prácticas de auto-atención más restringidas en herbolaria y apegadas a la medicina moderna. No obstante, al comparar los resultados obtenidos por niveles de escolaridad al interior del grupo que declara pertenecer a algún pueblo originario se observa que quienes tienen más años de estudio poseen pocos conocimientos sobre el sistema médico indígena, in-

26 Breilh, 2003: 47. 
cluyen fármacos en sus prácticas de auto atención y se vinculan más al sistema público de salud.

Por otra parte, la adscripción religiosa a las iglesias evangélicas incide en un distanciamiento de las formas de atención vinculadas a los yatiris, agentes de sanación para un tipo dolencias específicas, para centrarse en el pastor y a combinar tecnología médica andina con las creencias de estas opciones religiosas. Las personas que declaran pertenecer a esta opción religiosa ponen énfasis en la condición demoníaca y maligna de las antiguas divinidades. En cambio, quienes se adscriben a la iglesia católica tienden a acudir a los servicios del yatiri o waytiri, y en ocasiones, también al pastor evangélico ${ }^{27}$. Los medios de comunicación (radio y televisión) inciden tanto en los discursos sobre dietas alimenticias «saludables», como en el uso de fármacos ofrecidos en los publicitarios. Esto es más fácil de «consumir» entre las personas que tienen más contacto con el servicio de salud pública. Las entrevistas constatan argumentos como «una alimentación equilibrada» en base a frutas, verduras, yogur, leche, etc., y entre los medicamentos aparece el uso de dipirona, ibuprofeno y amoxicilina. De este modo, las formas de autoatención combinan la automedicación (medicinas de la industria farmacéutica), hierbas medicinales, medicinas elaboradas artesanalmente por los sanadores, por laboratorios peruanos y bolivianos en base a materias primas provenientes de la selva y rituales de sanación.

\section{LOS SABERES ACERCA DEL CUERPO Y SU FUNCIONAMIENTO}

En la base del concepto de salud se hallan las representaciones del cuerpo. Le Breton ha señalado que los saberes acerca de él son la expresión de un estado social y van transformándose a través del tiempo. En las sociedades modernas se produce una ruptura entre el sujeto y el cosmos, el cuerpo es la parte indivisible del sujeto, el factor de individuación. Las actuales concepciones del cuerpo están vinculadas con el ascenso del individualismo como estructura social y con la emergencia de las ciencias ${ }^{28}$. Si los pueblos indígenas son partícipes de los procesos de modernización, ¿Cómo se produce el proceso de separación entre el sujeto y el cosmos?

27 Este es el caso de la familia del joven que falleció producto de la enfermedad kajta o agarrado por seren t'alla. Los padres y abuelos decidieron «entregarlo» a Dios en la iglesia pentecostal antes de morir, a pesar de su firme decisión de permanecer entre los católicos.

${ }^{28}$ Le Breton, 1999. 
La secularización de los campos culturales y de las prácticas simbólicas ha ido más lento en la población indígena que en la no indígena. Ha ido avanzando a medida que la escolarización se expande, pero ésta se ha extendido de manera diferenciada según los grupos sociales que componen la población tarapaqueña. Es posible distinguir variaciones por estatus socio-económicos, por género y/o por pertenencia a las familias que descienden de la población indígena. El sistema escolar ha intervenido en el campo del conocimiento del cuerpo y su funcionamiento a través de la transmisión de los saberes de la biología. Por ello es que a mayor información y comprensión de las explicaciones que otorga esta disciplina, mayor es la separación entre cuerpo y cosmos. Entre quienes han cursado sólo enseñanza básica los saberes acerca de la dinámica corporal tiende a no separar cuerpo y cosmos, independientemente de su adscripción religiosa. Al mismo tiempo, se observa que en las personas con mayor escolaridad y/o con fuerte influencia de los medios de comunicación y residencia urbana permanente, se produciría la superposición de saberes entre los que la biología y el saber popular no indígena predominan sobre aquéllos que devienen de la historia de la medicina andina. En el otro extremo, entre las personas con menor escolaridad y con multiresidencia (rural-urbana) existiría un razonamiento positivo y religioso sobre la naturaleza y el universo; las concepciones del cuerpo tenderán a ser tributarias de la concepción de persona. La forma de concebir el proceso salud-enfermedad-atención se acerca a lo que han señalado otros autores para el sur andino.

La lengua aymara y chipaya actual no registra el término cuerpo, sólo se encuentra una aymarización de la palabra en español (kurpu). D. Arnold et. al. señalan que es difícil traducir el concepto de cuerpo en las lenguas andinas, lo cual se debería a que su epistemología no reconoce el concepto de un cuerpo distinto e individualizado. «No se piensa del cuerpo como algo que crece naturalmente sino algo producido por otros, en la acumulación corporal de conocimiento, sabiduría, alma, comida, etc. Por eso es más común utilizar conceptos como piel para describir la interfaz entre persona y ambiente. O se usan términos como qama para describir todo el complejo de nociones somáticas que engloban a la vida (ocupación, barriga, coraje, fuerza, sustento, descanso, placenta, y sangre menstrual) en una epistemología basada en los procesos de intercambio muy distinta de aquella de la medicina moderna» ${ }^{29}$. Sin embargo, se identifican lingüísticamente órganos articulados. En Tarapacá, la vida humana se compara anatómica y fisiológicamente a la vida animal, aun cuando se identifican ciertas particularidades. La comparación busca similitudes y dife-

29 Ibidem: 29. 
rencias, las que se asientan en una perspectiva de unidad en cuanto a lo esencial que une a estos dos seres (el ser humano y el animal); esto es que lo esencial está dado por la existencia — dentro del cuerpo - de un conjunto de órganos, relacionados entre sí, con ciertas funciones. Los matices diferenciadores entre unos animales y otros no escinden sino, por el contrario, interrelacionan, contribuyen a connotar una misma naturaleza, que es la que explica en todos los seres vivos el funcionamiento de los cuerpos concretos.

Se observa cierta categorización de un adentro y un afuera. Arnold y Yapita registran la distinción entre el cuerpo «físico» (lit.el «cuerpo superficial», patxa kurpu) y el cuerpo «espiritual» (lit. el cuerpo dentro manqha kurpu), que exige la aplicación de diferentes técnicas de curación, psicológica/ritual o físi$\mathrm{ca}^{30}$. No obstante, los antecedentes acopiados, hasta ahora, en nuestra región no confirman la asociación adentro = cuerpo espiritual, afuera = cuerpo físico. La parte interior, adentro, enfatiza en las vísceras cuya dinámica es lo más relevante para el funcionamiento del cuerpo. En aymara se llama lluqu al corazón propiamente tal. Existe consenso en que «el corazón» (chïma) está compuesto de tres partes; el bofe (pulmones), el hígado y el corazón. Éstos se vinculan a la cabeza, al cerebro por su relación con el pensamiento. La influencia de la biología se presenta en algunos casos a través de la duda, de la combinación de ideas. La persona de origen chipaya con enseñanza media completa, argumenta que el corazón es por la sangre no tanto con el pensamiento, luego reflexiona y formula que en el cerebro hay un pensamiento, un animu.

Los sanadores dicen que el corazón (lluqu), los pulmones (chïma), el hígado (k'iucha) son fundamentales para la vida, acercándose más a lo propuesto por J. Bastien. Este autor, sostiene que los andinos contemporáneos no creen en un alma o espíritu que sea inmaterial y esté agregado a sus cuerpos, tales como los conceptos post platónicos de un patrón ideal o las ideas cristianas de una forma inmortal. Ellos creen que el alma o ánima no es un ser, sino un fluido-físico, que a veces se describe como vapor o resuello. Nos informa que un análisis etnohistórico de las partes del cuerpo muestra que lo conceptualizan como principalmente constituido por un corazón (un sistema orgánico responsable de las funciones cognoscitiva, nutritiva y de bombeo) y samay, esto es un sistema orgánico responsable de la respiración. Argumenta que alma y animo /animu/ se utilizan el uno por el otro para referirse a las emociones, el vapor y el aliento que fluye a través del cuerpo. De este modo, concluye que los andinos conciben el cuerpo en términos de procesos orgánico y respiratorio que hacen circular sustancias vitales (sangre, alimentos, la respiración y

30 Ibidem: 28. 
las emociones). Estas sustancias son físicas, aunque no se puedan ver directamente ${ }^{31}$.

G. Fernández, por su parte, señala que el corazón (chuyma), es considerado uno de los órganos más importantes del cuerpo humano. Este sería el centro de la persona, puesto que en él se ubicarían las tres sombras o las tres almas: $m \ddot{a}$ ауи (primer ajayu) о Ајауu, pä ајауu (segundo ajayu), о ánimu, kimsa ajayu (tercer ajayu) o coraje. Agrega que el corazón es un lugar inespecífico dentro del organismo que facilita el contacto con los principales órganos corporales del ser humano, potenciando el intercambio de fluidos corporales. En el corazón se ubica todo lo relativo al ánimo, como también las vísceras y las entrañas del cuerpo, es decir, lo que está dentro del individuo ${ }^{32}$.

Los andinos de Tarapacá coinciden en que el cuerpo humano y animal está compuesto por un elemento central para su funcionamiento: el animu. También se le puede llamar ispiritu. Se trataría de un componente material e inmaterial para el buen funcionamiento del organismo. Se puede ubicar en todo el cuerpo, aunque también se reconocen dos localizaciones específicas: la cabeza y el corazón.

La reproducción humana se representa en el cuerpo femenino en la figura de la matriz. En el cuerpo masculino su equivalente es el padre (asociado en términos modernos a la próstata). El embarazo se percibe en el cuerpo femenino cuando «salta la matriz». La placenta presenta una connotación de peligro para la madre si queda dentro del cuerpo y debe enterrarse luego del parto. Se observan diferencias en los cuerpos femeninos y masculinos. Argumentan que los hombres son de fierro y las mujeres de barro: las mujeres se gastan más luego. Por eso los hombres se demoran más en criarse. Las partes más sensibles en el hombre son la próstata, los riñones, pulmones, dolores de músculos o huesos. Se enferman por el frío, el consumo de alcohol, la comida salada. Las mujeres es algo parecido, pero lo más sensible es la matriz. Ellas se enferman del cerebro, corazón, recaída de la matriz, los riñones, matriz pasada de frío. Su estómago es más firme que el del hombre, sus huesos se vuelven blandos por amamantar, pérdida de calcio. El trabajo sexual hace debilitar a los hombres por pérdida del semen.

El cuerpo sano es aquél en el que la circulación de la sangre es adecuada. No todas las personas son iguales en este sentido, lo que se observa, empíricamente, en la temperatura corporal y ésta se percibe en la vena palpando el pulso e interpretando su condición a través de tres categorías: chuñuri (fría),

\footnotetext{
31 Bastien, 1986: 8.

32 Fernández Juárez, 2004: 283.
} 
junt'u, cálida, junt'uptat (tibio). La otra sustancia esencial es la grasa. J. Bastien sostiene que la distribución de la grasa es, junto a la circulación de la sangre, a la unión de un fluido aglutinante (animu) y a la eliminación de fluidos tóxicos, los componentes básicos para la salud ${ }^{33}$.

Nuestra interpretación de los datos sostiene que chïma expresaría un concepto clave para comprender la naturaleza del cuerpo humano. Si los órganos se especifican a través de términos lingüísticos propios, chïma no podría equivaler a corazón propiamente. La relación que se establece entre los órganos principales (lluqu, k'iucha, chïma) y a la acción de sentir-pensar nos dice que se trataría de un concepto complejo, de un nivel de abstracción mayor. Esta idea puede sostenerse también si analizamos otros campos semánticos, como el que se observa en la textilería o en la concepción del territorio. Chïma sería el centro del espacio textil tripartito, configurando un cuerpo animal, con referencia a su interior (adentro y profundo ${ }^{34}$. Otra analogía utilizada para la representación del cuerpo en la que aparece el concepto de chïma se encuentra en la concepción del espacio que habitan. Éste se concibe como la anatomía del cuerpo humano concebido en tres partes unidas orgánicamente a través de las entrañas, chïma ${ }^{35}$.

Otra lectura posible es que si chïma nombra a los pulmones ${ }^{36}$, el centro de las cosas y de las personas, la función que cumple este «órgano» para dar vida se relacionaría con el sistema respiratorio y el aliento vital como dimensión principal del animu (ver infra). Una interpretación como ésta plantea la cuestión de por qué se traduce y o define como corazón. Se puede suponer que fue y es una equivalencia realizada para comprender la idea de la centralidad del corazón para la medicina colonialista. Sea una u otra idea la más acertada, podemos decir que la estructura orgánica del cuerpo (de los cuerpos) opera sobre procesos que van más allá de lo estrictamente biológico, garantizando la armonía general del todo corporal. Se desprende una determinada fisiología del cuerpo, con base en una condición energética (motor) y en una correcta coordinación y sincronía de sus segmentos o partes constitutivas.

\section{EL ANIMU ELEMENTO SUSTANTIVO E INMANENTE DE LA VIDA}

La comprensión de los fundamentos ontológicos y epistémicos de la teoría de la vida para los andinos no secularizados pasa por entender el concepto de

33 Bastien, 1986: 14.

34 Cf. Cereceda 33/5-6 (Paris, 1978): 1030.

35 Gavilán Vega, 30 (Arica, 2005): 140.

36 García Choque, 2005: 36. 
апітu. Es una parte del cuerpo cuya naturaleza no es fácil de entender. Sólo una interpretación ligera podría argumentar que este concepto equivale al de ánimo, al de alma y/o al de espíritu; no obstante sus estrechas relaciones. Contiene referencias materiales e inmateriales y se halla en la base del modelo etiológico-terapéutico del sistema médico indígena.

\section{ACERCA DEL CONCEPTO DE ANIMU}

Es posible identificar dos aproximaciones a los contenidos de este concepto. Una de ellas sostiene que el animu sería un componente del organismo de las personas, animales y vegetales, de las entidades animadas (fuentes de agua, montañas) cuya naturaleza es material e inmaterial al mismo tiempo. La otra se inclina por enfatizar en su inmaterialidad.

La Riva es una de las autoras que ha investigado especialmente sobre sus significados. A partir de data etnográfica de la región del Cuzco y de fuentes etnohistóricas concluye que el animu sería una «fuerza anímica» no sólo humana. También recibe el nombre de ispiritu y sería la entidad que da vida al cuerpo (principio vital). Una vez que la persona fallece, se denomina alma al animu del muerto, y se encuentra íntimamente asociado al aya (cadáver). Esta autora constata que no hay una concepción única ni unívoca de animu, sino una pluralidad de representaciones y una variación en el número de entidades anímicas que involucra tanto diferencias regionales, como de género. Varía a lo largo del ciclo de vida de un individuo en un proceso paulatino de adquisición de fuerza anímica y fijación de la misma en el cuerpo para decrecer con la edad hasta la pérdida total con la muerte. Respecto de su localización, argumenta que este se encuentra repartido por todo el cuerpo; no obstante, un análisis más detallado muestra que el animu se puede concentrar en ciertas zonas y sustancias corporales: en el «corazón»sunqu, en la «cabeza» uma, en la «sangre» yawar, así como en el mismo nombre de la persona, entre otros. Agrega que el animu es también identificado o comparado con la sombra de un individuo, que aparece como su manifestación visible. Se identifica con sonidos o formas de animales alados como las mariposas, luciérnagas, moscas o pajaritos, especialmente cuando los sanadores lo reintegran al cuerpo a través de un tratamiento terapéutico ${ }^{37}$.

Hemos visto que J. Bastien marca la dimensión material del animu. Cita a Oblitas para recuperar la idea de fluido que da consistencia al cuerpo: «Él quiere

37 La Riva, 41 (Cuzco, 2005): 64. 
decir que es un fluido que psicológicamente liga todo el cuerpo. El animo se llama juchuy ajayu (ajayu pequeño) y el alma se llama jatun ajayu (grande). Cuando una persona pierde su ajayu pequeño se enferma, se desorganiza y su cuerpo empieza a secarse (la sangre se vuelve delgada, la piel empalidece, se manifiesta una extenuación general). Cuando una persona pierde su jatun ajayu, la persona muere, porque su cuerpo se seca ${ }^{38}$. Dicho de otro modo, agrega: «Los qollahuayas (sic) consideran al animo y al alma como fluidos que fluyen a través del cuerpo, en forma análoga a la energía eléctrica que es invisible, pero que conecta todas las partes con una sustancia cargada. Ellos distinguen el animo del alma, en que el animo es utilizado para describir la partida temporal de este fluido, pero el cuerpo está todavía en el ciclo húmedo y seco. En otras palabras, el fluido puede retornar al cuerpo seco. El alma se refiere al fluido que se separa del cuerpo en el momento de la muerte, de suerte que el cuerpo permanece en el ciclo seco y el alma en el ciclo húmedo. El animo y el alma no se consideran seres sino fluidos dentro de los ciclos húmedo y seco del cuerpo» ${ }^{39}$.

Entre aquéllos que tienden a considerar que se trataría de un componente inmaterial, hallamos a G. Fernández, a Arnold y Yapita y a X. Albó. Fernández señala que entre los campesinos aymaras del área de Jesús de Machaqa, así como los del sector lacustre de Santiago de Huata y Achacachi (provincia Omasuyo, departamento de La Paz) las entidades anímicas que poseen los seres humanos en el altiplano reciben la denominación de ch `iwi, «sombra», y son tres (kimsa ch `iwi) las entidades que poseen la característica de ser sombras gemelas entre sí y con respecto a su propietario, por cuanto constituyen su «doble». Cada una de estas tres «sombras» recibe una denominación específica: la primera y más importante es conocida como ajayu, término antiguo recogido por Bertonio ${ }^{40}$ que lo define como «la sombra de todas las cosas». El ajayu es la sombra principal, su pérdida implica la muerte irreparable del doliente. Las otras «sombras» reciben denominaciones prestadas del castellano; tal es el caso del animu, «animo», de carácter secundario frente a la primera. La tercera de las «sombras» recibe la denominación de kuraji, «coraje» y su pérdida resulta solo un episodio sin importancia». En el cruce semántico de sombra y animu, atribuye a las sombras una existencia dual: por un lado, en el interior de la persona, en su corazón, chuyma, por otro, en la «sombra» externa, ch'iwi, sobre la que se aprecia el contraste de matices que la luz provoca entre el ajayu interno y el «coraje» externo ${ }^{41}$.

38 Oblitas 1963: 32-33, en Bastien, 1 (Perú, 1986): 13.

39 Ibidem: 13.

40 Bertonio Ludovico, 1612/1984, II: 108.

41 Fernández Juárez, 2004: 279-280-281. 
Arnold y otros definen el término ajayu como sinónimo de ispiritu y como lo que va junto con la persona, cuidándola como «ángel de la guarda». Dicen que el animo o espíritu suele dejar el cuerpo de la parturienta durante el parto dejándoles sin fuerzas ${ }^{42}$. Por su parte, X. Albó nos dice que la palabra espíritu, que trató de imponerse a los andinos, sólo se usa en contextos muy específicos: para referirse al Tata Espíritu, pero no en una identificación con el espíritu santo, sino como un día dentro del ciclo agrícola, el «día de pentecostés» en que se realiza la fiesta de los productos recién cosechados y se marca el inicio de la estación del «hambre». El otro uso de espíritu, lo cual equivale a animu, es para referirse a un arquetipo germinal, el aliento vital. Según este autor existe una distinción básica entre los términos animu y alma: animu se refiere al espíritu de lo vivo, mientras que alma se utiliza más para referirse al de los muertos. Existirían tres espíritus vitales que se clasifican según su capacidad de desprenderse del cuerpo: estos son animu, ajayu y coraje. El elemento que permite referirse a los tres es "chiwi», vale decir sombras, tanto en el sentido de la sombra material proyectada por los cuerpos, pero también en el sentido de todo lo que anteriormente se llamaba supay (demonios). Estos tres espíritus pueden abandonar el cuerpo incluso en vida, generando enfermedades, por lo cual se requiere rituales capaces de devolverlos a su lugar ${ }^{43}$.

En un estudio sobre la concepción de la vida y la muerte en Isluga, norte chileno, M. Ortega se refiere a la existencia de tres almas. Alma sería equivalente a los contenidos asignados al de animu o ajayu por estos autores, pero los significados difieren toda vez que estos serían exclusivamente metafísicos y desvinculados de su naturaleza material. Sin embargo, es posible que las referencias a las ideas cristianas impuestas para evangelizar tengan alguna relación ${ }^{44}$.

\section{El CONCEPTO DE ANIMU EN TARAPACÁ: Fragmentos de la Historia}

Los significados identificados no son unívocos en los entrevistados. Coinciden en la importancia superlativa que le asignan al animu en el bienestar de las personas. No se registró el término ajayu. Los sanadores explican que el cuerpo incluye tres sombras que nos acompañan siempre, ch'iju. Un animu. Cuando las personas mueren el animu se convierte en alma. Éstas no se ven,

42 Ibidem: 57.

43 Albó, 2000.

44 Ortega Perrier XXXIII/2 (Arica, 2001): 255. 
sólo se sienten. La señora chipaya argumenta «es como el ángel de la guarda que tiene uno». Las personas que no están bautizadas arriesgan a que su animu sea llevado por los gentiles o chullpas, especialmente cuando tienen hambre. Estos no son cristianos y están asociados a mallku-t'alla, deidades masculina y femenina, que protegen y dictan las normas, la ética. Una persona con buen comportamiento protege su animu. Aquél que rompe las reglas corre el riesgo de perder el animu. Si el animu se escapa, por acción de gentiles y demonios-divinidades con quienes comparten la vida, afecta especialmente al pensamiento y provoca circulación alta. La posibilidad de la muerte o de enfermarse en forma crónica e irrecuperable (como volverse loco, p.ej) depende de la capacidad del sanador (yatiri o waytiri) para hacer retornar el animu al cuerpo; mientras más tiempo pasa o más lejos se va el ánimu, se reducen las posibilidades de curación, ya que puede ser capturado definitivamente por los $m a-$ lignos. Subyace una concepción de «urgencia crítica» respecto de estas formas de enfermar, en las cuales la oportunidad de intervención del yatiri es clave para un pronóstico.

El animu viene de la tierra (pacha), se va al cielo cuando la persona muere, pero no se va de inmediato, viaja, no se transforma en alma automáticamente, es un viaje costoso, que requiere de esfuerzos. Por otra parte, se trata de un elemento que recorre el cuerpo: Sube o baja dentro del cuerpo, se sube al cerebro o baja al corazón. Su función, es el pensamiento, la capacidad de razonar, de permitir una buena circulación de la sangre y de aquí al bienestar. Sin animu, la persona se vuelve tonta. Pisi chïma, en Isluga significa poca inteligencia ${ }^{45}$. La utilización del concepto «pensamiento» admite la posibilidad de entenderlo como un sinónimo de «conciencia», se necesita de la conciencia para que uno tenga ánimo (estado de ánimo). Pero, ella se hallaría articulada a «las entrañas».

El razonamiento no abstrae la materia, al contrario, enfatiza en la intrincada materialidad de la vida. Chïma, término equivalente a chuyma en la lengua aymara que se habla en la región de Arica y Bolivia se halla en el diccionario de Bertonio con muchas acepciones Una de ellas dice: «los bofes propiamente, aunque se aplica al corazón y al estómago y a casi todo lo interior del cuerpo». Y que chuyma amajasitu significa acordarse; y, memoria, como potencia del alma se nombra como Chuyma amajasiña, amutaña. El pensamiento también requiere o se posiciona en el corazón, por ejemplo, cuando uno se enamora; sin el corazón funcionando no puede haber pensamiento, uno piensa con el cerebro pero también con el corazón. El animu está vinculado a la cabeza, al ce-

45 García Choque, 2005: 36. 
rebro, al corazón, no tanto en términos de órganos sino en términos de relación: una ligación que se expresa en el pensamiento.

La acción de consolar se expresa en Isluga como chimacht'aña ${ }^{46}$. Chuyma churasiña, nos dice Bertonio significaba poner su corazón o pensamiento en alguna cosa; chuyma «todo lo perteneciente al estado interior del [animo], bueno o malo, virtud o vicio, según lo que le precediere». De este modo, su registro identifica aspectos relacionados al carácter de las personas y/o a estados de ánimo (soberbio, terco, enojadizo, amar, suspiros de mozos por su enamorado) y a la imaginación.

La idea del «carácter» entendido como proceso que se cultiva social y «biológicamente» está presente en el concepto de ciclo vital; lo cual se relaciona con lo señalado por La Riva. En Isluga, el curso de la vida se representa como un proceso que exige varios ritos de pasaje: bautizos, matrimonio, pasante de las fiestas patronales, cacicazgo ${ }^{47}$. Las exigencias sociales que otorgan prestigio y dan centralidad a la persona (Jaqui) se relacionan con la pertenencia a una comunidad. Esta condición sólo se logra con el matrimonio. De aquí en adelante se debe cultivar el corazón, el pensamiento ${ }^{48}$ a través del cumplimiento de las costumbres. Una persona que ha cumplido correctamente a lo largo de su vida con el ideal de una persona tiene «corazón» y exitosamente cultivado. Al contrario, un niño no tiene «corazón», los jóvenes lo están formando ${ }^{49}$. Los jóvenes suelen enfermar de pensamiento cuando no son correspondidos en el amor. Se trata de una fase crítica en la formación personal. La pérdida del animu es más fácil en los niños y jóvenes que en los adultos, estos tienen mayor capacidad de razonar, mayor inteligencia; es más fácil en las personas cuyos comportamientos éticos no son correctos (el corazón para alguno, el cerebro para otros). Dicen las abuelas, relata una mujer, que estas tienen siete pensamientos y los hombres solo dos, argumento que se acerca a la información recogida en Cuzco, donde se considera que las mujeres poseen más animu que los hombres, dándose la cifra de siete para el género femenino y de tres o uno para el masculino» ${ }^{50}$.

46 Ibidem: 118.

47 Las fiestas que celebran el término de los estudios de enseñanza media adquieren un carácter similar.

48 Bertonio propone que Jaquikanka significaba la persona, existencia del hombre (humanidad).

49 Gavilán Vega, 30 (San Pedro de Atacama, 2005): 142.

50 La Riva, 41 (Cuzco, 2005): 82. 


\section{LA VIDA-El CUERPo: La SALud-LA ENFERMEDAD}

Es posible argumentar que el pensamiento indígena sostiene que el desequilibrio de la temperatura afecta la salud provocando diferentes padecimientos. Una aproximación certera al enfermo es aquélla que da cuenta de su condición cálida, fría o cálido-fría. Es por ello que se insiste en el examen físico a través de la palpación del pulso y se mira con desconfianza el proceder de algunos médicos que no se dignan en examinar a sus consultantes. No procede a la manera del exorcismo alopático intentando erradicar de un solo golpe todo mal, sino pausadamente acompañar al enfermo en su recuperación facilitando el proceso mórbido en su curso.

Las sanadoras y los sanadores expresan su conocimiento del funcionamiento del cuerpo a través del ritmo de la circulación de la sangre en las venas, lo cual se relaciona con el corazón chïma-animu. Al parecer lo que las personas ingieren afecta a la sangre, como sustancia y a la circulación. Una mala circulación de la sangre afecta a todo el organismo. Las pastillas e inyecciones mal administradas cortan o interrumpen la circulación de la sangre, produciendo «encogimiento», algo así como parálisis del cuerpo. Interrumpe procesos biológicos básicos y en algunos casos puede producir la muerte. La idea de la circulación intracorpórea como un proceso en el que sustancias y fluidos se desplazan por un sistema de tubos o canales internos impregna gran parte de la explicación del enfermar, del desplazamiento interno de la enfermedad y, por tanto, de su localización final en la topografía o anatomía del cuerpo. La sangre, que circula por las venas hacia todo el cuerpo, partiendo del corazón, es el elemento capital; su curso es posible seguirlo con una palpación fina siguiendo el trayecto de los vasos sanguíneos de la superficie, también las características de grosor, fuerza, velocidad de sus pulsaciones clarifican aspectos diagnósticos centrales. Fluyen o se desplazan a modo de circulación o de tránsito interno otros componentes: la respiración y sus secreciones, los alimentos por el lumen digestivo, la menstruación por el aparato genital, fluyen sensaciones y corrientes por el nervio. Se enferma todo aquello que presenta una obstaculización de dicho tránsito: alimentos y elementos extraños que tapan las tripas; las secreciones bronquiales que se estancan y no fluyen, la pérdida del flujo menstrual por enfriamiento de la matriz o por embarazo, etc. Subyace aquí una representación muy dinámica de flujos dentro de sistemas o circuitos internos pensados según una lógica de equilibrio interno en el que el concepto de cálido, frío, cálido-frío opera de manera importante. De este modo, la medicina andina se esfuerza por eliminar todo lo que entorpece los flujos, saca sustancias tóxicas del cuerpo, favorece la circulación de la sangre restableciendo equilibrio frío/calor, limpia el interior del tubo digestivo obstruido, masajea el 
nervio doliente, etc. En esta parte de su tarea el médico aymara se asemeja a la imagen del médico del sistema de salud: en tanto sujeto con cuyos conocimientos está interviniendo directamente sobre determinados órganos y procesos internos con el fin de establecer la cura de la enfermedad; éste es el momento del acto médico en el cual se analizan síntomas, se explora irregularidades, se prescriben tratamientos, como hace el doctor.

La tendencia a buscar equivalencias para comprender los diferentes pensamientos médicos está presente también en los sanadores. Algunos de ellos intentan comparar su propia perspectiva anátomo-fisiológica con la que podría mostrarle la moderna imagenología del cuerpo que ofrece la ciencia médica. Buscan las imperfecciones que a esta última le impide reconocer la simplicidad con la que ellos se explican ciertos procesos internos como el parto, por ejemplo.

Desde su modelo médico explicativo se entienden estos procesos de manera similar a lo que la sociedad moderna entiende como fisiología. Sin embargo, las correspondencias que el pensamiento andino establece entre el cuerpo (individual-colectivo) el territorio y el cosmos nos hablan de un modelo relacional. En consecuencia, sería un error realizar esta equivalencia pues el modelo anátomo-fisiológico que subyace en la medicina moderna naturaliza al cuerpo humano; es decir, lo separa de su realidad social. Si bien es cierto los sanadores apuntan a explicaciones «fisiológicas», se diferencian del positivismo en que no pierden la conciencia de totalidad. Estas relaciones son posibles por la fuerza interna, que es la que está comprometida en la interioridad de estos procesos, y que es la responsable en última instancia de la vida humana. Fuerza interna vinculada al cosmos a través del animu. La vida sería un proceso que el médico aymara reconoce y describe en la materialidad del cuerpo, como condición necesaria pero no suficiente, pues la humanidad se debe a las generaciones anteriores (achachilas, mallkus-t'allas), quienes dictaron las normas y ética del buen vivir. Las generaciones pasadas, presentes y futuras se integran en lo material e inmaterial, inscritos en un territorio tan conocido como un cuerpo viviente, todas forman parte de un universo en el que la vida humana no se escinde de aquello que la hacen posible.

\section{REFLEXIONES FINALES}

Hemos planteado que un camino para el estudio de los saberes para la salud y la enfermedad y de las prácticas médicas en la sociedad y cultura andinas que considere la perspectiva histórica debería situar la investigación empírica en el contexto del proceso salud-enfermedad-atención experimentado por los 
grupos sociales concretos. En este marco ha sido posible acceder a información valiosa que nos informa que un punto de articulación entre la medicina andina tarapaqueña y la biomedicina es la equivalencia que existiría del cuerpo como conjunto de órganos relacionados entre sí que cumplen determinadas funciones. En ambos casos la materialidad del cuerpo humano y su funcionamiento están siempre presentes en los diagnósticos y en los pronósticos. En este sentido, las clasificaciones realizadas en términos de enfermedades mágico-religiosas exclusivamente referidas a causas sobrenaturales simplifican algunas de las prácticas médicas identificadas aquí. Si bien la religiosidad invade el campo de la salud en la mayoría de los grupos sociales como una forma de aliviar la incertidumbre, observamos que entre los aymaras menos secularizados, a diferencia del cristianismo en su forma hegemónica, asignan materialidad a las fuerzas metafísicas.

La restauración de la salud promueve diversas estrategias de atención, cuya lógica es conciliar las diferentes formas de concebir la vida y la muerte en un contexto en el que la confianza suele ponerse entre paréntesis hasta obtener resultados concretos. En el caso de S. podríamos adelantar una respuesta, igualmente tentativa. La celeridad que exige el tratamiento de la huida del ani$m u$, producto de la coyuntura - debilidad del joven y la época en que las entidades del pasado (Supay) vuelven - no se pudo cumplir por dos razones. Por una parte, sus padres desconocían las andanzas del hijo adolescente, enamorado, con escaso conocimiento de los lugares peligrosos del altiplano. Por la otra, no había en ese momento un especialista para acudir. El agravamiento de S. llevó a sus padres a buscar solución en la medicina moderna, pero ésta no pudo satisfacer sus necesidades. Las explicaciones al desarrollo de la enfermedad se dieron, silenciosamente, por vías paralelas. Los equipos de profesionales del sistema de salud público realizaron una lectura unilateral del problema. No comprendieron las interpretaciones que la familia de S. realizaba de su padecimiento, impidiendo un eventual encuentro de saberes puestos en tensión. Es probable que los neurólogos encargados no se informaran del desenlace final del proceso mórbido: la muerte del joven. Esta situación grafica la distancia real con que intervinieron unos sanadores y otros en este proceso. Por otra parte, el no seguimiento del caso, una vez dado de alta y la carencia de un concepto claro de continuidad de cuidados y cuidados compartidos, propios del modelo comunitario en salud, refleja la ausencia de recursos humanos y materiales con que opera el equipo de salud para asegurar este seguimiento clínico y bajo la perspectiva de colaboración intercultural. En un contexto en el que las políticas neoliberales se manifiestan en un proceso de reformas sanitarias privatizadoras, resulta aún más difícil enfrentar los problemas de salud de los grupos subalternos. 
En tanto, la comunidad étnica reafirmaba sus convicciones: S. falleció engullido por Seren T'alla, entidad peligrosa particularmente por su voracidad, por su «hambre-deseo» de sangre y grasa, llevando consigo su animu. Extrajo la fuerza vital de S., como si Seren T'alla estuviese premunida de una intensa materialidad, tal como la tuvo S.

Este hecho removió la conciencia de las personas que se estaban distanciando de estos razonamientos para profundizar la tensión entre las argumentaciones del sistema médico andino y las de la biomedicina. Pero esta tensión va desapareciendo a medida que las personas adhieren a las explicaciones de la biología y por lo tanto han separado cuerpo y cosmos. Así, podríamos señalar que las continuidades y o «la fidelidad cultural» en salud tienden a estar presente en el grupo que ha participado escasamente en el sistema público de educación. De aquí que el campo de la salud no es en sí mismo un espacio privilegiado para estudiar la cultura andina en Tarapacá. De acuerdo a los datos acopiados, un lugar apropiado para el estudio de las continuidades culturales es el grupo de menor escolarización al interior de la colectividad de origen indígena o entre aquellos que han tardado en incorporarse a la modernidad; pues poseen un gran bagaje de conocimientos, tecnologías y experiencias legadas por sus antepasados, pero también acumuladas por su propia agencia. Son los agentes comunitarios para la salud en sus diversas especialidades, quienes exponen saberes provenientes de diferentes fuentes y continúan experimentando nuevas estrategias de sanación para ofrecer sus servicios a un sector de la población de origen indígena. Si bien es preciso continuar la investigación empírica sobre este sistema médico particular, no se debería dejar de lado el estudio del proceso de transformaciones; específicamente los saberes y prácticas médicas en aquel segmento con mayor escolaridad a fin de observar el proceso de acercamiento a la biomedicina y el alejamiento del modelo médico andino. La focalización en uno u otro grupo es importante para contar con mejores herramientas para promover el respeto por un tipo de conocimiento y experiencia milenaria y el controvertido diálogo intercultural, pues en el mejor de los casos este sigue siendo una aspiración o un deseo y en el peor de ellos, sólo otra estrategia para continuar con la relación de dominación cultural del modelo biomédico promovida por el sistema de salud público.

Con todo, las prácticas médicas seguidas por la población con ascendencia indígena en la región continúan aliviando malestares y sanando enfermedades reales o imaginarias, es decir entregando soluciones a problemas de salud que no son resueltos por otras propuestas médicas; pero sobre todo, proporcionando sentido de pertenencia a una colectividad, fortaleciendo sus vínculos con el pasado para comprender el mundo moderno. 


\section{BIBLIOGRAFÍA}

Albó, Xavier, Preguntas a los historiadores desde los ritos andinos actuales, trabajo presentado al encuentro Cristianismo y Poder en el Perú Colonial, Cuzco, Fundación Kuraka, 2000. http://albo.pieb.com.bo/archivos/Preguntas_Xavieralbo.pdf

Arnold, Denise, Juan de Dios Yapita y Margarita Tito, Vocabulario aymara del parto y de la vida reproductiva de la mujer, La Paz, Instituto de Lengua y Cultura Aymara y Family Health International, 1999.

Augé, Marc, «L'anthropologie de la maladie», L’Homme 26/97 (Paris 1986): 81-90.

Bastien, Joseph, «Etnofisiología Andina: evidencia lingüística, metafórica, etiológica y etnofarmacológica para conceptos andinos sobre el cuerpo», Arinsana / 1 (Perú 1986): 5-24.

Bastien, Joseph, «Kallawaya-Andean Body Concepts», en Healers of the Andes. Kallawaya Herbalists and their Medicinal Plants, University of Utah Press (Salt Lake City 1987): 67-76.

Bertonio, Ludovico, Vocabulario de la lengua aymara, CERES/IFEA/MUSEF, Cochabamba, 1984 [1612].

Breilh, Jaime, Epidemiología crítica. Ciencia emancipadora e interculturalidad, Buenos Aires, Lugar Editorial, 2003.

Cereceda, Verónica, «Semiologie des tissus andins: les talega d'Isluga», Annales 33/5-6 (Paris 1978): 1017-1035.

Citarella, Luca (comp.). Medicinas y culturas en la Araucanía, Santiago, Editorial Sudamericana, 1995.

Comelles, Josep María y Ángel Martínez Hernáez, Enfermedad, Cultura y Sociedad, Madrid, EUDEMA, S.A. (Ediciones de la Universidad Complutense, S.A.), 1993: 85 .

Cuche, Denys, La noción de cultura en las ciencias sociales, Buenos Aires, Ed. Nueva Visión, 2002.

Chamorro, Andrea y Constanza Tocornal, «Prácticas de salud en las comunidades del Salar de Atacama: hacia una etnografía médica contemporánea», Estudios Atacameños, 30 (San Pedro de Atacama, 2005): 117-134.

Fernández Juárez, Gerardo. «“Ajayu, animu, kuraji”. La enfermedad del "susto” en el altiplano de Bolivia», Gerardo Fernández (comp.), Salud e Intercultiuralidad en América Latina. Perspectivas antropológicas, Quito, Ecuador Ediciones Abya-Yala, 2004: 279-303.

Foster, George y Barbara Anderson, Medical Anthropology, Nueva York, Alfred A. Knopf, 1978.

Freidson, Eliot, La profesión médica, Barcelona, Ediciones Península, 1978. 
García, Pedro, Aru Pirwa, Diccionario Aymara-Castellano y Castellano-Aymara, Iquique, Consejo Nacional de la Cultura y las Artes, 2005.

Gavilán Vega, Vivian, «Representaciones del cuerpo e identidad étnica en la población indígena del norte de Chile», Estudios Atacameños, 30 (San Pedro de Atacama, 2005): 135-148.

Gavilán Vega, Vivian, «Festividades andinas y religiosidad en el norte chileno», Chungará, 41/1 (Arica, 2009): 84-101.

Guerrero Jiménez, Bernardo, «Medicina andina y medicina pentecostal en los aymaras del norte grande de Chile: del yatiri al pastor», Chungara, XXVII/2 (Arica 1995): 153-165.

Gundermann Kroll, Hans, Comunidad, sociedad andina y procesos socio-históricos en el norte de Chile, Tesis para optar el grado de Doctor en Ciencias Sociales con Especialidad en Sociología de El Colegio de México, 2001: 11-490.

Gundermann, Hans y Héctor González, «Sociedades indígenas y conocimiento antropológico: aymaras y atacameños de los siglos XIX y XX», Chungará, 41/1 (Arica, 2009): 113-164.

Grebe Vicuña, María Ester, «En torno a los ritos terapéuticos astrales de Isluga», Chungara, 10 (Arica 1983): 155-164.

Grebe Vicuña, María Ester, Culturas indígenas de Chile: un estudio preliminar, Santiago, Editorial Pehuen, 1998.

Kessel, Juan van, «La Medicina andina», Cuadernos de Investigación Social, 13 (Iquique 1985).

Kessel, Juan van, «Ayllu y ritual terapéutico en la medicina andina», Chungara, 10 (Arica 1983): 165-176.

Kleinman, Arthur, Patients and healers in the context of culture: An exploration of the borderland between anthropology, medicine and psychiatry, Los Angeles, University of California Press, 1980.

Larraín, Jorge, Modernidad razón e identidad en América Latina. Santiago de Chile, Editorial Andrés Bello, 1996.

La Riva González, Palmira, «Las representaciones del animu en los Andes del sur peruano», Revista Andina, 41 (Cuzco 2005): 63-861.

Le Breton, Antropología del Cuerpo y Modernidad, Buenos Aires, Editorial Nueva Visión, 2006.

Menéndez, Eduardo, «La enfermedad y la curación ¿Qué es la medicina tradicional?», Alteridades 4/7 (México D.F. 1994): 71-83.

Menéndez, Eduardo, «Modelos de atención de los padecimientos: de exclusiones teóricas y articulaciones prácticas», Ciencia \& Saúde Colectiva, 8/1 (Rio de Janeiro 2003): 185-207. 
Ortega Perrier, Marietta, «Escatología andina: metáforas del Alma», Chungara, XXXIII/2 (Arica 2001): 253-258.

\section{AGRADECIMIENTOS}

Agradecemos a los yatiris y qolliris y a las amigas y amigos que compartieron sus conocimientos, sus reflexiones e inquietudes; por su acogida en los patios de sus hogares, entre montañas majestuosas.

Fecha de recepción: 29-12-2009

Fecha de aceptación: 11-11-2010

\section{Contemporary Andean society and culture: a study of the knowledge of health and disease in the indigenous villages of northern Chile}

The study of the knowledge of health and disease and of medical practices among the people of indigenous origin raises theoretical, social and political challenges. From the analysis point of view, their cultural heterogeneity makes it necessary to consider the modernization process. Said process involves taking a historical perspective that is not always taken into account in research on contemporary Andean society and culture. This paper seeks to move in this direction. We propose that the weight of schooling is gravitating towards social and cultural differentiation and therefore affects the level achieved in understanding the nature of the body and how it works, as well as health practices. The data gathered largely coincides with that reported by other researchers. Based on this, we propose that it is necessary to extend the debate on the issue of materiality-immateriality of the theoretical foundations of Andean medical thought.

KeY words: Contemporary Andean Society and Culture; Andean Medicine; Indigenous knowledge; Health and Indigenous Peoples. 